\title{
GAIT REHABILITATION USING THE HUBER 360 PLATFORM IN MULTIPLE SCLEROSIS
}

\section{Carmen Liliana GHERGHEL ${ }^{1 *}$, Mariana CORDUN¹, Gigi TEODORU ${ }^{1}$, Lucian Călin MARIN²}

\author{
${ }^{1}$ National University of Physical Education and Sport, Faculty of Kinetotherapy, Bucharest, Romania \\ 2 "Dr. Chiriac" Medical Rehabilitation Centre, Bucharest, Romania \\ *Corresponding author: carmen_gherghel@yahoo.com
}

DOI: $10.35189 /$ iphm.icpesk.2019.12

\begin{abstract}
Multiple sclerosis (MS) is the most common demyelinating disease of the central nervous system, representing the second cause of disability of young adults after trauma. Approximately $85 \%$ of patients show either relapsing-remitting forms or secondary-progressive forms. The treatment mainly aims to rehabilitate functions after stroke, prevent new strokes and prevent disability. Numerous studies have emphasised that the medical recovery process of MS patients has short-term positive influence on joint mobility, balance and other aspects of the quality of life. The physiotherapy protocol includes assessing and addressing the body's ability to function, focusing in particular on walking and mobility, strength, balance, posture, fatigue and pain. The Huber 360 software can be used for assessment purposes; seven tests can be run on this platform, and, in this paper, we have used the tests related to walking. Huber 360 is also intended for therapeutic use, given that it includes programmes aimed at muscle strength, range of motion or obesity-associated therapy. The purpose of this research is to determine the impact of using the Huber 360 platform on gait rehabilitation in patients with multiple sclerosis. The study included three subjects, one woman and two men. Platform results for the gait test show that the subjects have improved their walking, which has been materialised by an increased number of steps.
\end{abstract}

Keywords: multiple sclerosis, physiotherapy, Huber 360.

\section{Introduction}

Multiple sclerosis (MS) is a progressive, chronic form of primitive demyelinating encephalomyelitis. This disease mainly affects young adults and is very rarely encountered in children (Drăgănescu et al., 1962). In 1866, Charcot described for this pathology the presence of a triad consisting of: nystagmus, intentional shaking, jerky voice and plantar cutaneous reflex in extension (cerebellar, vestibular and pyramidal phenomena) (Aisen, Sevilla, \& Fox, 1996). In 1926, Pierre Marie issued the hypothesis that MS was infectious, and Bullok issued the viral (infectious) hypothesis, which was supported by Levaditti in 1930 and Margulis, Subladeze and Gaidamovici in 1948 (Ionel, 1997, p. 129).

Nowadays, MS is considered a complex disease, with at least four identified pathological types found by Lucchinetti et al. (2000) and four distinct developments (Braddom, 2015, p. 1301).

Generally, multiple sclerosis is progressive and worsens at a variable pace according to an uncertain pattern. A weekly fluctuation may occur as exacerbations are replaced by remissions. Exacerbation may occur at any time, the distinctive sign of this disease being the uncertainty concerning its future progression.

Numerous studies have shown that the medical recovery process of patients diagnosed with MS has short-term positive influence on the functional independence and implicitly the quality of life. In general, the most impressive positive influences have been highlighted by uncontrolled retrospective clinical studies including patients going through the post-exacerbation recovery stage (Braddom, 2015, p. 1301).

Studies trying to find an extended transfer effect have shown that, without monitoring therapy, the positive influences disappear after 6 to 10 months. This finding was attributed to disease progression in certain stages, while disease progression was not a possible explanation in other stages, the Expanded Disability Status Scale (EDSS) remaining stable.

Generally, studies have addressed the effects of conventional physical rehabilitation programmes on the spacetime walking parameters at regular and high speeds. The participants were distributed according to both their walking speed and the criteria applied to people with stroke and MS (Perry, Garrett, Gronley, \& Mulroy, 1995). These studies have shown that the forming effects can be present at both normal and high speeds, the most obvious effects being highlighted by people walking at low speed (Carmela et al., 2018).

When comparing walking performance to walking speed (normal or faster speed), it was found that the parameters that had been improved at normal speed had also been improved at higher speed in the case of the disadvantaged group (Motl et al., 2012). These results support the treatment of patients with moderate to severe walking difficulties, who have limited acceleration capability (Kesselring, 2001). 
According to the type of movement, it has been found that MS people who move at lower speeds show significant improvements from a clinical point of view and in terms of the space and time parameters: speed, double support and step length (Snook \& Motl, 2009).

No other neurological condition impacts so many functions of the central nervous system. At the moment, the MS management has as guidance a better understanding of the disease and the awareness of its effects on the family life, friends, professional activities and communities.

The purpose of the medical recovery treatment for MS patients can be difficult to achieve. Contrary to the changing agents of the disease, the state of the patients tends to worsen over time.

In general, studies suggest that people with MS who have recently shown signs of walking difficulties and have gone through a moderate-intensity programme consisting of combinations of exercises are proof that this approach represents a rehabilitation strategy associated with improved walking (Scheinberg et al., 1980).

\section{Material and Methods}

Multiple sclerosis is a condition with progressive development; its progression rate and development are variable. Studies highlight a number of people with confirmed MS who satisfactorily manage their daily activities for a long time, sometimes years, without treatment. MS only slightly affects the quality of life of these people. On the other hand, there are people whose disease, even following intensive therapies, progresses rapidly and they do not survive its various complications (bed sores, pneumonia and kidney failure) (Braddom, 2015, p. 1310).

In people with MS, impaired walking may be caused by contracture, loss of muscle strength, spasticity, fatigue, proprioception disorders, cerebellar or vestibular dysfunctions, visual impairments or the inability to multitask. About one third of people diagnosed with MS show a certain degree of walking impairment (Barkhof et al., 2003).

Compared to healthy people, patients with MS show reduced step length, slower walking speed, shorter rotation of the hips, knees and ankles (stiff walking), etc. Generally, these people take small quick steps and do not have full range of motion (Braddom, 2015, p. 1310).

In MS people, the orthosis plays a major role in optimising walking. Therefore, it is important for patients to be prescribed ankle-foot orthoses to optimally improve their movements with a tolerable amount of comfort. The reduced strength of hip flexors is commonly encountered in MS, and recently an orthosis has been designed to manage this problem, namely the hip-flexing support orthosis, which can be useful in improving limb movements.

Specific gait rehabilitation, along with the use of an assistance device and an adequate orthosis for a MS person is a challenge for any physical therapist. Studies suggest that non-specified exercise programmes, as a component of ambulatory treatment, are inefficient for improving gait (Braddom, 2015, p. 1311).

To this end, we believe that the monitored multiaxial Huber $360 \mathrm{MD}$ platform is a modern and successful method used for gait rehabilitation in MS people. This device is the result of many years of research and combines the latest technologies, perfection and reliability with LPG Systems, leaders in the field.

Huber 360 MD must only be used by professionals. For therapeutic purposes, it can be used to increase muscle mass, regain range of motion and as an obesity-associated therapy. This software can also be used for patient assessment; this option can be accessed through the reporting mode to perform diagnosis tests and generate patient assessment. The device must be connected to a tablet to use this feature.

The Huber 360 MD platform allows to applying 7 assessment tests: stability, unipodal stance, gait, stability limits, mobility restriction, maximum resilience and coordination. Each of these tests is controlled using the tablet. Also, the results will only be visible on the tablet.

According to the study purpose and objectives, out of the 7 tests, we shall only use the gait test.

Based on the previous arguments, the purpose of this paper is to highlight that using the Huber 360 platform in the medical rehabilitation protocol of patients with multiple sclerosis determines the improvement of walking, with an impact on increasing the quality of life of these patients.

The study involved three subjects, one woman aged 29 and two men aged 35 and 50, respectively. To include them in the study, we used inclusion and exclusion criteria. The inclusion criteria were: remissive-type multiple sclerosis, Expanded Disability Status Scale (EDSS) < 4.5 and ability to move; the total score was defined by the functional scores, evolution within 1 to 8 years, last spurt about 1-2 years ago. The exclusion criteria were: progressive/recurrent multiple sclerosis forms, main and secondary progressive forms, Expanded Disability Status Scale (EDSS) $>5$ patients with movement difficulties and are granted equivalents of the functional scores $<2$ years $>8$ years, final spurt $<1$ year. 
The physiotherapy program on the Huber 360 platform was developed throughout 10 sessions, for two thirds of the sessions, at a rate of one meeting per week. At the onset of fatigue or any type of discomfort, the meeting was interrupted.

For the physiotherapy programme on the Huber 360 platform, two types of exercises were selected: for posture and balance, on the one hand, and to increase physical endurance, on the other hand, all of this falling in line with the objectives pursued. These exercises had a relatively low difficulty in order to avoid fatigue or stress in patients, which would not have been recommended for our simple specific therapeutic approach.

A very important aspect in the execution of exercises was that the subjects had the opportunity to watch their own performance, having visual feedback on the platform's monitor and thus being able to adjust their movements for better compliance with the task requirements.

\section{Results}

The results obtained in the tests performed at the initiation of the medical rehabilitation protocol using the Huber 360 platform (initial testing) and two months later using the physiotherapy application of the Huber 360 software (final testing) indicated that the patients have improved their gait. The research subjects had a recurrentremissive form of MS, and the result analysis has shown that their age (woman: 29, men: 35 and 50, respectively) and form of disease cause significant variations in results; the disability status also causes a significant variation in the number of steps.

Table 1. Results for the gait test on the Huber 360 platform

\begin{tabular}{lccc}
\hline \multicolumn{1}{r}{ Parameters } & Initial & Final & Difference \\
\hline S1 & & & \\
Number of steps & 65 & 69 & 4 \\
Acquisition time & 50 & 50 & - \\
S2 & & & \\
Number of steps & 72 & 72 & - \\
Acquisition time & 50 & 50 & - \\
S3 & & & \\
Number of steps & 68 & 74 & 6 \\
Acquisition time & 50 & 50 & - \\
\hline
\end{tabular}

The results in Table 1 show that all subjects have achieved satisfactory results. Two subjects made good progress increasing their movement abilities; thus, they managed to increase the number of steps in 50 seconds with a difference of 4 and 6 steps, respectively. The third subject managed to maintain the number of steps (70 steps in 50 seconds) under medication-induced discomfort conditions. It is worth mentioning that, when examining gait, the information received from several systems (visual, vestibular, cerebral, motor ones and more) should be taken into consideration.

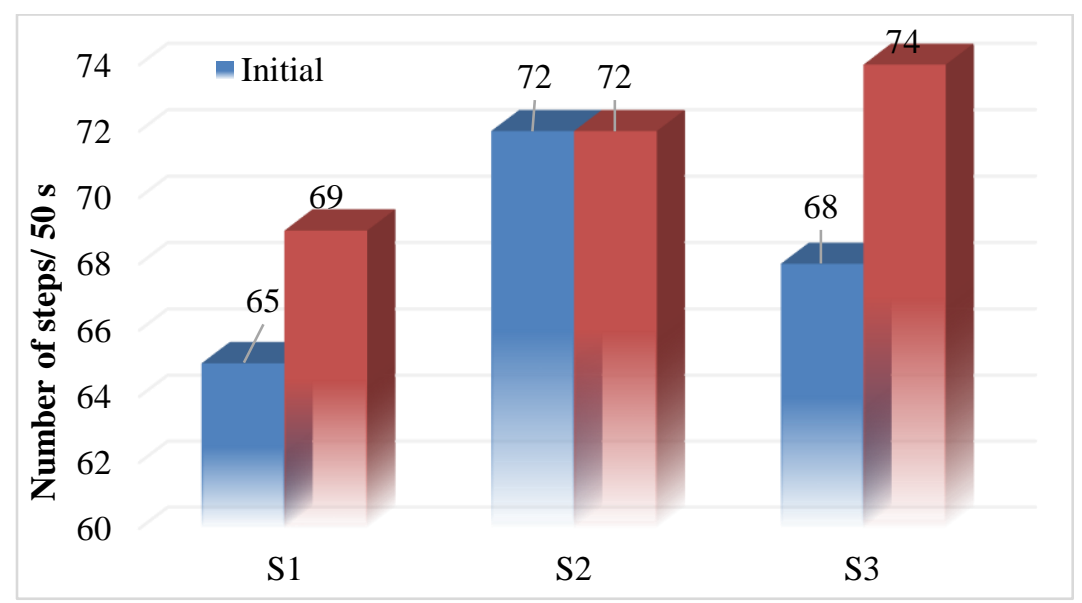

Figure 1. The gait test (S1 - subject 1, S2 - subject 2, S3 - subject 3) 
The cause of gait dysfunction can be determined by understanding the complications that might occur during disease progression. Figure 1 shows that two subjects have obtained an increase in the number of steps in 50 seconds. Subject 2 managed to maintain 70 steps in 50 seconds under the discomfort conditions induced by medication (Avorex); he was aged 50 years, had an EDSS score of 4, and the onset of his disease had occurred 7 years ago. Subject 3 managed a considerable improvement in the number of steps in 50 seconds, which was reflected in the difference between the initial and final tests, namely 6 steps; he was aged 35 years, had an EDDS score of 2.5 and the onset of his disease had occurred 4 years ago.

\section{Conclusion}

The results of gait tests on the Huber 360 platform show that all subjects have achieved satisfactory results. Two subjects made good progress increasing their movement abilities; thus, they managed to increase the number of steps in 50 seconds, and the third subject managed to maintain the number of steps in 50 seconds under medication-induced discomfort conditions.

The training on the Huber platform, in addition to the overall muscular activity, allows the rehabilitation of proprioception and balance in a specific way. The stimulation of physical and motor skills was also performed.

It is worth mentioning that, when examining gait, the information received from several systems (visual, vestibular, cerebral, motor ones and more) should be taken into consideration.

Exercises performed on the Huber 360 platform have a beneficial effect on disability and the quality of life of people with multiple sclerosis. This might be due to the fact that patients can mobilise enough muscle mass to obtain a real effect of the training.

\section{References}

Aisen, M. L., Sevilla, D., \& Fox, N. (1996). Inpatient rehabilitation for multiple sclerosis. Neurorehabilitation and Neural Repair, 10(1), 43-46. https://doi.org/10.1177\%2F154596839601000106

Barkhof, F, Bruck, W, De Groot, CJ, Bergers, E., Huslhof, S., Geurts, J., ... van der Valk, P. (2003). Remyelinated lesions in multiple sclerosis: Magnetic resonance image appearance. Archives of Neurology, 60(8), 1073-1081. https://doi.org/10.1001/archneur.60.8.1073

Braddom, R. L. (2015). Medicină fizică şi de reabilitare [Physical and rehabilitation medicine] (Ed. a IV-a). Bucureşti: Elsevier.

Carmela, L., Kalron, A., Smeda, T., Normann, B., Wens, I., Eijnde, O. B., \& Feys, P. (2018). Effects of rehabilitation on gait pattern at usual and fast speeds depend on walking impairment level in multiple sclerosis. International Journal of MS Care, 20(5), 199-209. https://dx.doi.org/10.7224\%2F1537-2073.2015-078

Ionel, C. (1997). Compendium de neurologie: Anatomofiziologie, patologie clinică, tratament [Compendium of neurology: Anatomy-physiology, clinical pathology, treatment]. Bucureşti: Editura Medicală.

Kesselring, J. (2001). Rehabilitation in MS is effective. International MS Journal, 8(2), 68-71.

Lucchinetti, C., Brück, W., Parisi, J., Scheithauer, B., Rodriguez, M., \& Lassmann, H. (2000). Heterogeneity of multiple sclerosis lesions: Implications for the pathogenesis of demyelination. Annals of Neurology, 47(6), 307-717. https://doi.org/10.1002/1531-8249(200006)47:6\%3C707::aid-ana3\%3E3.0.co;2-q

Motl, R. W., Smith, D. C., Elliott, J., Weikert, M., Dlugonski, D., \& Sosnoff, J. J. (2012). Combined training improves walking mobility in persons with significant disability from multiple sclerosis: A pilot study. Journal of Neurologic Physical Therapy, 36(1), 32-37. https://doi.org/10.1097/NPT.0b013e3182477c92

Perry, J., Garrett, M., Gronley, J. K., \& Mulroy, S. J. (1995). Classification of walking handicap in the stroke population. Stroke, 26(6), 982-989. https://doi.org/10.1161/01.str.26.6.982

Scheinberg, L., Holland, N., Larocca, N. G., Laitin, P., Bennett, A., \& Hall, H. (1980). Multiple sclerosis: Earning a living. NY State Journal of Medicine, 80(9), 1395-1400.

Snook, E. M., \& Motl, R. W. (2009). Effect of exercise training on walking mobility in multiple sclerosis: A metaanalysis. Neurorehabilitation and Neural Repair. 23(2), 108-116. https://doi.org/10.1177/1545968308320641 